\title{
Editorial introduction to the special issue on stochastic matching models, matching queues and applications
}

\author{
Jean Mairesse ${ }^{1} \cdot$ Pascal Moyal $^{2}$
}

Accepted: 21 November 2020 / Published online: 8 February 2021

( ) The Author(s), under exclusive licence to Springer Science+Business Media, LLC part of Springer Nature 2021

In the current boom of online services, internationally leading companies promote applications that offer their users an interface to interact and collaborate. We can think of the users as members gathered in classes, which use the application as an interface to find a match. Matching mechanisms are also useful in many other physical systems, subject to compatibility constraints between tasks, and in healthcare systems such as blood transfusions and organ transplant networks.

All the aforementioned applications share a clear common ground: the arrivals of requests are random, and their sojourn in the system is settled upon finding a match (a date for next Saturday, a compatible kidney, a qualified cook for next evening, etc.), that is identified as such according to fixed compatibility rules. In all cases, the system controller aims to optimize the waiting time of the requests before finding a match, to control the traffic and avoid congestion, or to design a pricing algorithm, for instance. We have thereby defined a particular class of skill-based queueing systems, in which the compatibility graph plays a central role together with the traffic constraints.

Formally introduced in the pioneering paper of Caldentey, Kaplan and Weiss (2009), stochastic matching models have been primarily viewed as a variant of skillbased service systems in which customers and servers play symmetric roles: server come and go in the system, exactly like customers, and use the system as an interface to find a match. In subsequent works, central questions on this class of models have been addressed, among them stability conditions, control for fairness or end-toend delay, and the derivation of characteristics at equilibrium. Recent development in the study of such systems also included:

Jean Mairesse

Jean.mairesse@lip6.fr

Pascal Moyal

Pascal.moyal13@gmail.com

1 CNRS, LIP6, Sorbonne Université, 75005 Paris, France

2 Institut Elie Cartan de Lorraine - UMR 7502, Université de Lorraine, Site de Nancy, B.P. 70239, 54506 Vandoeuvre-lès-Nancy Cedex, France 
- the derivation of product-form formulae for the stationary state under the matching policy 'First Come, First Served,'

- a generalization of the compatibility constraints to general, instead of bipartite, graphs,

- the construction of optimization tools for models under general compatibility constraints.

Many open and intriguing problems remain to be solved regarding stochastic matching models. In particular, the criteria for the stability or the optimality of given matching algorithms remain graph-dependent, and a solution for general graph geometries is still missing. Likewise, the very form of the stationary state under general control policies (other than FCFS) is unknown. We can continue this non-exhaustive list of open problems with the extension of such models to the context of reneging, or with the optimization of matchings algorithm using statistical learning procedures, among others.

We believe that this class of models will constitute an active and fruitful avenue of research for the years to come, for it is, at the same time, a model of high practical relevance for applications and of deep mathematical complexity.

Motivated by this very active line of research, we are pleased to guest edit this special issue of Queueing Systems, Theory and Applications on Stochastic matching models, Matching queues and applications. We have selected two high-quality papers (out of six submissions) that best illustrate the current trends of research in the field:

In Matching Queues with Reneging: a Product Form Solution, Francisco Castro, Hamid Nazerzadeh and Chiwei Yan derive explicit product-form solutions for the steady state distributions of an ' $N$ ' system in which customer renege. For doing so, the authors obtain remarkable balance equations that are peculiar to the case of abandonment and shade an interesting light toward a generalization of the aforementioned approach of product forms for matching systems.

In Directed FCFS Infinite Bipartite Matching, Gideon Weiss considers an original asymmetrical variant of the bipartite matching model, in which goods and agents are matched in FCFS order, but goods that do not find an available agent upon arrival are immediately lost (with immediate applications to car/ride sharing, organ transplants and two-sided web markets). The author derives crucial performance metrics, such as the matching rates and end-to-end delays. Interestingly, simple algorithms are provided for computing the normalizing constants-another crucial feature in the context where the steady state is characterized under a product form.

Publisher's Note Springer Nature remains neutral with regard to jurisdictional claims in published maps and institutional affiliations. 